\title{
Inducing large deformation in wood cell walls by enzymatic modification
}

\author{
Luna Goswami - Michaela Eder · Notburga Gierlinger • \\ Ingo Burgert
}

Received: 10 April 2007/Accepted: 11 September 2007/Published online: 4 December 2007

(C) Springer Science+Business Media, LLC 2007

\begin{abstract}
It has been shown recently that wood with a high cellulose microfibril angle in the S2-layer, e.g. compression wood, shows permanent plastic deformation without significant mechanical damage to the matrix. This molecular stick-slip mechanism was explained by a gliding of the cellulose fibrils, after a certain shear stress in the matrix was exceeded [1]. Such a material behaviour would be desirable for various applications, for instance, to cover complex geometries with highly deformable veneers as needed in the automotive industry. However, veneers that are typically used for these purposes have a rather brittle failure behaviour, which leads to breakage, and drastic quality and productivity losses. A better deformability of such veneers might be achieved when the underlying deformation principles are conferred by modifying the wood cell wall components, in particular the cellulose fibrils and their matrix coupling. Enzyme treatments were performed on mechanically isolated wood fibres to plastify the entire lignified secondary cell wall. Cellulase Onozuka R-10 from Trichoderma viride (E.C.3.2.1.4) with activity on cellulose and xylan was utilized. Micromechanical tests and FT-IR microscopy studies revealed the change of mechanical properties and nanostructural features of the cell wall. An extended deformability was achieved for two of ten of the modified fibres.
\end{abstract}

\section{Introduction}

A key concept in nature is the embedding of stiff particles or fibres in a pliant matrix. Some of the biological materials

L. Goswami · M. Eder · N. Gierlinger · I. Burgert ( ()

Department of Biomaterials, Max-Planck-Institute of Colloids

and Interfaces, Potsdam 14424, Germany

e-mail: ingo.burgert@mpikg-golm.mpg.de are mineral-polymer composites, such as bone or nacre, others, such as plant cell walls or tendon, are polymerpolymer composites. Their extraordinary mechanical properties originate from the structural organization and chemical interaction between the components based on a strong coupling with interface polymers [2]. By this means, materials of both high stiffness and high toughness are created even in mineralized systems, which are anticipated to be rather brittle such as nacre [3] or the deep sea sponge Euplectella [4]. Hence, the nanostructural organization of biological systems can function as a source of bioinspiration for the design of new engineering materials [2, 5-7], and/or a better understanding of the underlying principles can be used to modify the natural material to design new "bio-based" systems with tuned mechanical properties.

The native wood cell wall is a complex and highly optimized fibre composite structure. It consists of the three bio-polymers cellulose, hemicelluloses and lignin. The parallel oriented cellulose is a semicrystalline homopolymer which forms units of $\sim 2.5 \mathrm{~nm}$ thickness, the so-called microfibrils. These are embedded in the pliant matrix polymers, in which hemicelluloses have a specific mediating and coupling function. E.g. in softwood where the hemicellulose glucomannan is proposed to be associated with the cellulose fibrils and xylan with lignin [8]. The orientation of the cellulose microfibrils predominately dictates the mechanical behaviour of the cell wall [9-11]. An almost parallel orientation of the cellulose to the cell axis (low microfibril angle) results in a stiff but rather brittle material, whereas a high mircofibril angle leads to a less stiff but tough material [11] (Fig. 1). It was recently shown for wood with a high cellulose microfibril angle (compression wood), that this material could undergo permanent plastic deformation without significant mechanical damage of the matrix. This molecular stick-slip 


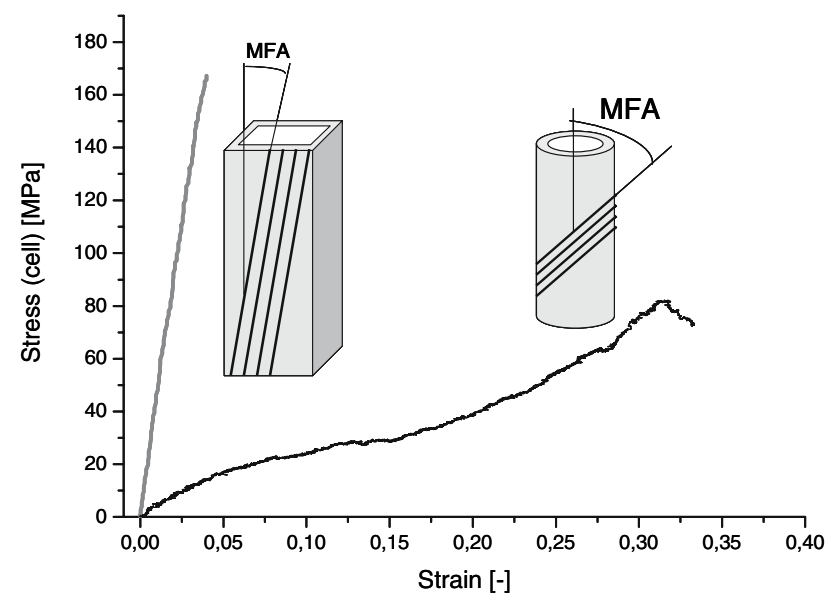

Fig. 1 Comparison of stress-strain curves of an earlywood (grey line) and a compression wood fibre (black line) tested in axial tension in the wet condition. The stress was calculated on the basis of cell cross-sectional area. Schematic drawing of cell shape and cellulose microfibril angle (MFA)

mechanism beyond the yield point can be explained by a gliding of the cellulose fibrils, after the shear strenght of the matrix is exceeded [1]. Such a material behaviour would be desirable for various applications, in particular for covering complex geometries with highly deformable veneers. However, commonly used veneers are made of normal wood tissue with a small cellulose microfibril angle, which leads to cracking and buckling when complex geometries are veneered [12]. This substantial and costintensive problem might be solved, by utilizing compression wood for the veneer process [13]. However, mainly availability and optical aspects interfere with this kind of substitution.

An alternative approach is to modify the molecular structure of the commonly utilized material and induce sustained gliding of microfibrils due to a severe alteration of the fibre-matrix composition. A shortening of the aligned fibrils and a loosening of the interface between the stiff elements and the pliant matrix may alter the cell wall in a way that even normal wood becomes less stiff and shows extended plastic deformation beyond yield. In a basic study at the individual fibre level, we treated lignified cell walls with a multicomponent enzyme attacking both cellulose and xylan. Single fibre tensile tests and FT-IR microscopy were utilized to mechanically and chemically characterize the cell wall modification.

\section{Materials and methods}

From never dried normal wood of spruce (Picea abies [L.] Karst.) $200 \mu \mathrm{m}$ thick tangential sections were cut using a rotary microtome. Single wood fibres were mechanically isolated under a light microscope by peeling them out of the transition wood part between early- and latewood using very fine tweezers [14]. Single fibres were utilized to access the individual cell wall and rule out interfering effects by cell-cell interactions due to their tight interfaces. For enhancing the accessibility of enzymes, both ends of the fibres were cut with a sharp razor blade. Finally, isolated fibres were vacuum dried for $5-10 \mathrm{~min}$ by using a desiccator.

The single fibres were treated with enzyme after a controlled swelling (260 $\mu \mathrm{L} / 10$ fibres) in lithium chloride$N, N$-dimethylacetamide $(0.7 \mathrm{~g}$ of $\mathrm{LiCl}$ soluble in $10 \mathrm{~mL}$ of DMAC), which was performed for 2-3 days at room temperature. Preliminary tests had shown that a pretreatment of a cell wall is needed and that the described swelling protocol can provide penetration of enzymes even in the presence of almost unaltered lignin [15]. After swelling, fibres were washed three times with water for regeneration. Proper protective care was taken during handling of all the chemicals, particularly DMAc and $\mathrm{LiCl}$ to avoid toxicity. Fibre modification was performed using the multicomponent enzyme "Cellulase Onozuka R-10 from Trichoderma viride (E.C.3.2.1.4)" (Yakult Pharmaceutical Industry, Co. Ltd, Japan), which so far has been used for milled wood and pulp modification [15]. Cellulase Onozuka contains endoglucanase, exoglucanase, $\beta$-D-glucosidase and xylanase. Endoglucanase is known to break the main backbone, while exoglucanase is known to break the end chains of cellulose. In contrast, $\beta$-glucosidase acts on cellobiose to produce glucose. Xylanase is responsible to break the main backbone of xylan.

Enzyme of a concentration of $0.1 \mathrm{~g} / \mathrm{mL}$ was dissolved in buffer (50 mM Na $2 \mathrm{HPO}_{4}$ titrated with Citric acid, $\mathrm{pH} \mathrm{5}$ ). The wood fibres were incubated with mechanical shaking at $50^{\circ} \mathrm{C}$ up to 3 days. Cellulase acts on $\beta-1,4$-glycosidic bonds and produces cellobiose or glucose as end products, which act as competitive inhibitors for the cellulase and result in feedback inhibition [16]. To overcome this feedback inhibition, enzyme solution was changed frequently (at least 6 times).

The result of enzymatic treatment was evaluated by micromechanical tests and Fourier transform infrared (FTIR) microscopy on modified and untreated reference fibres. Micromechanical investigations were done in the wet state by coating the fibres with glycerin prior to testing. For details on the testing procedure see [17]. For acquiring FTIR spectra, single fibres were placed on $\mathrm{KBr}$ windows and air-dried at room temperature. An FT-IR microscope (Hyperion 2000) equipped with a liquid nitrogen cooled MCT detector and connected to a Vertex 70 FT-IR spectrometer (Bruker $\mathrm{GmbH}$, Germany) was used. A total of 128 scans were co-added per sample spectrum (wavenumber range: $4,000-700 \mathrm{~cm}^{-1}$ ) and apodized, applying 
the Blackman-Harris three-term function and a zero filling factor of 2 . Three spectra were acquired along each fibre with an aperture of $100 \mu \mathrm{m} \times 30 \mu \mathrm{m}$ and averaged.

\section{Results}

Swollen wood fibres without enzymatic treatment reveal mechanical properties similar to normal wood fibre properties (Fig. 2a, b). But a statistically significant change in mechanical properties was observed when the swollen fibres were further treated with the enzyme. After enzyme treatment all fibres showed a lower strength and stiffness than the reference ones. After reaching the ultimate stress level, enzymatically treated fibres behaved in two different ways: $80 \%$ of the fibres revealed a brittle fatal fracture at much lower forces than the normal wood fibres (Fig. 3a), but about $20 \%$ of the fibres showed a partial fracture with a further high plastic deformation up to $20 \%$ (Fig. 3b). This relationship confirms preliminary results from numerous tests (data not shown here).

For control, fibres were treated with heat-inactivated enzymes and buffer only. No change in the mechanical
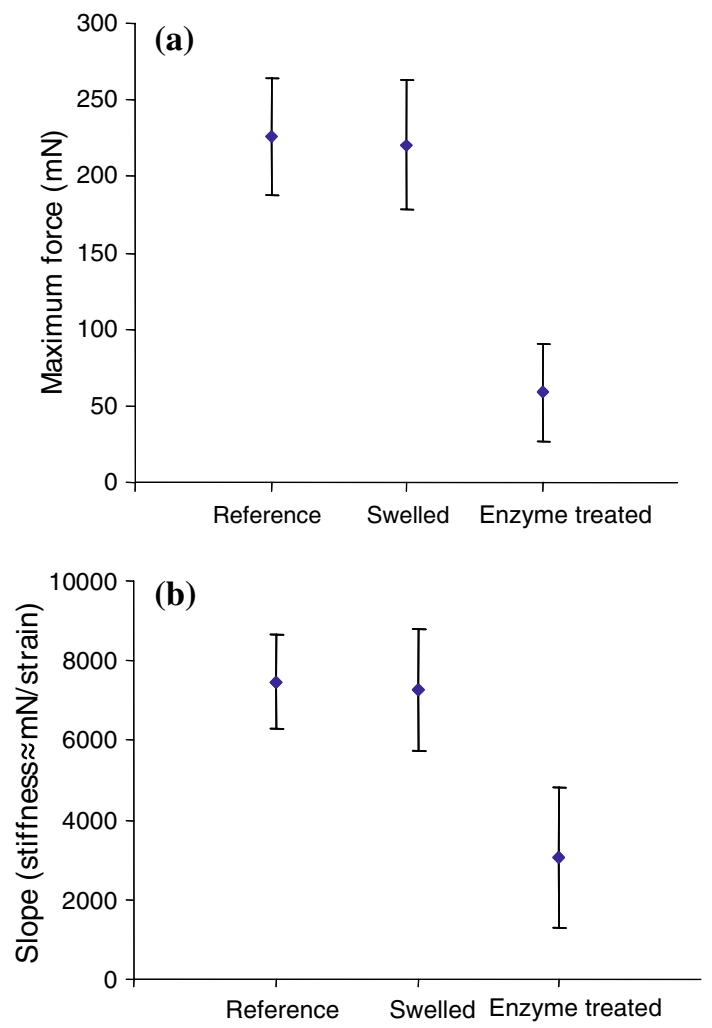

Fig. 2 Comparison of the mechanical properties of untreated reference fibres $(n=10)$, swelled fibres $(n=10)$ and enzymatic treated fibres $(n=10)$. (a) Maximum force (in $[\mathrm{mN}]$ units) required for breaking the fibres. (b) Slope ("stiffness" $\approx[\mathrm{mN} / \mathrm{strain}]$ ) of the force-strain curve. The error bars show standard deviation
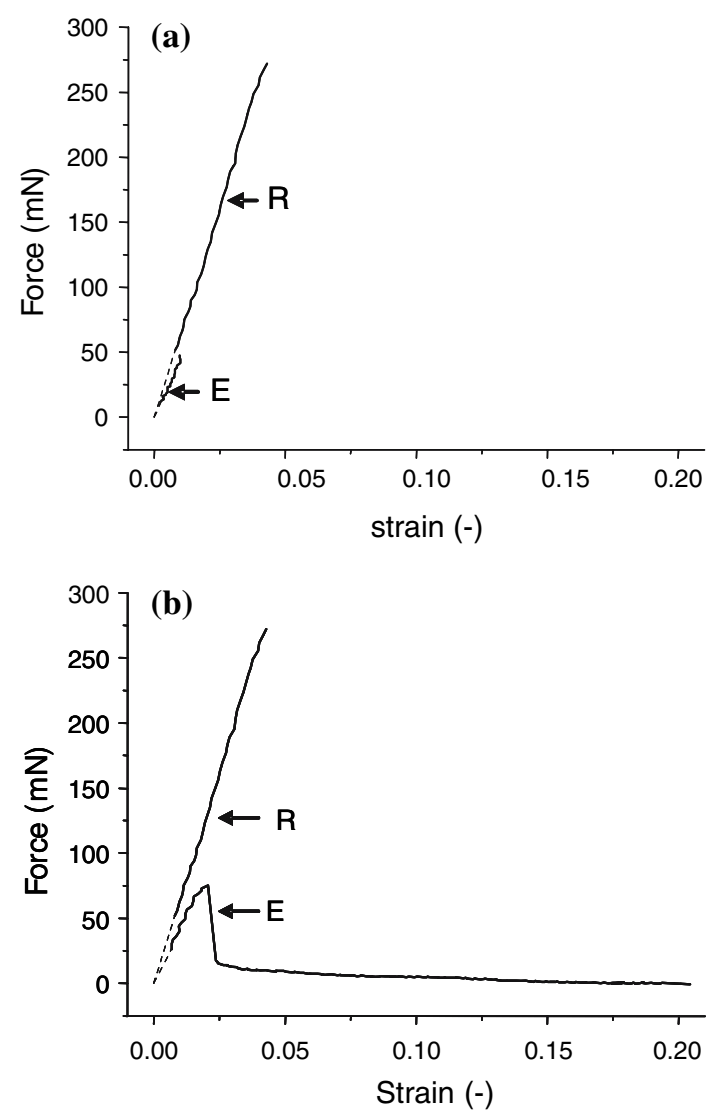

Fig. 3 Force-strain diagrams of mechanically isolated fibre (R) and enzymatically treated fibre (E). (a) Eight out of ten fibres break in much lower force than that of normal wood fibre. (b) The other two fibres show high deformability after a sharp yield point. For plotting fibres with an unsteady initial stage, the point of origin was determined by extrapolating the slope of the linear range (dotted line)

properties was observed when the fibres were either treated with heat-inactivated enzymes or buffer only.

To analyze the enzymatic modification at the molecular level, FT-IR spectra were acquired on the single fibres in the native state and after enzyme treatment with previous swelling. To exclude variability and changes due to different sample thickness or composition, exactly the same fibres were measured before and after treatment. Absorbance of all observed peaks was diminished in the enzyme treated fibres compared to the reference fibres (Fig. 4a). A reduction in the broad band around $3,368 \mathrm{~cm}^{-1}$, attributed to $\mathrm{OH}$ stretching, was observed as well as a reduction of the band at $2,900 \mathrm{~cm}^{-1}$, assigned to $\mathrm{CH}$ stretching (Fig. 4a) [18]. Beside these bands, which allow no specific conclusion on the changes of the different wood polymers, also the bands in the fingerprint region $\left(1,800-750 \mathrm{~cm}^{-1}\right)$ were less intense. Bands characteristic for carbohydrates, e.g. at $1,426 \mathrm{~cm}^{-1}$ (primarily due to $\mathrm{CH}_{2}$ scissor motion in cellulose) [19], at $1,160 \mathrm{~cm}^{-1}$ (asymmetric $\mathrm{C}-\mathrm{O}-\mathrm{C}$ bridge stretching [20]), at $1,108 \mathrm{~cm}^{-1}$ (ring stretching) and bands between 1,100 and $950 \mathrm{~cm}^{-1}$ (CO stretching [20]) were 

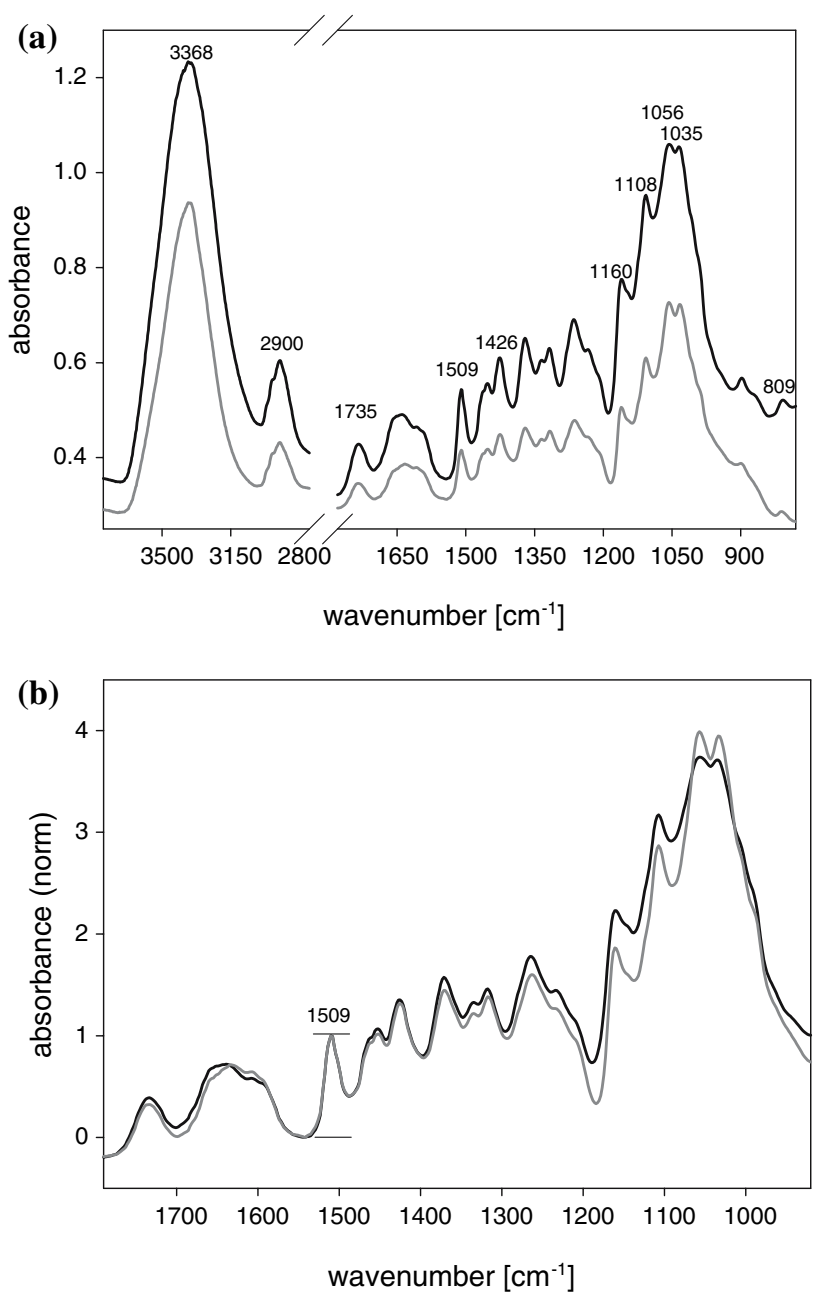

Fig. 4 (a) FT-IR spectra of mechanically isolated untreated control spruce fibres (black line) and enzyme treated fibres (grey line). Absorbance for the enzyme treated fibre is arbitrary as the curve was shifted downwards to minimize overlap of the bands. (b) FT-IR spectra in the fingerprint region after normalization on the lignin band at $1,509 \mathrm{~cm}^{-1}$

reduced. Beside the reported bands influenced by cellulose and hemicelluloses, the band at $1,735 \mathrm{~cm}^{-1}$ derives mainly from contributions of xylan ( $\mathrm{C}=\mathrm{O}$ stretching), and the band at $809 \mathrm{~cm}^{-1}$ from glucomannan [20, 21]. Both are also reduced in the enzyme treated fibres (Fig. 4a), indicating also a removal of xylan and glucomannan. However, we also observed a reduction of the characteristic lignin band at $1,509 \mathrm{~cm}^{-1}$ (specific for aromatic skeletal vibration [22]) suggesting partial removal of lignin. To see more clearly if all three wood polymers were diminished to a similar extent, spectra were normalized on the lignin band at $1,509 \mathrm{~cm}^{-1}$, as this sharp band represents solely the aromatic lignin structures. After normalisation most of the bands show similar heights (Fig. 4b), from what we conclude that all three wood polymers have been removed to a similar extent. The carbohydrate bands between 1,185 and
$900 \mathrm{~cm}^{-1}$ appear somehow sharper, which might indicate that more amorphous cellulose and/or hemicelluloses than crystalline cellulose was removed. Band positions changed only slightly $\left(1-2 \mathrm{~cm}^{-1}\right)$, concluding that the remaining wood polymers themselves are not severely changed, but the bonding environment might be slightly different.

\section{Discussion}

Enzymatic treatments can be used to modify biopolymers and their interaction, however, so far the entire cell wall of solid bulk wood samples have barely be modified by enzymes. Lignin significantly inhibits enzymatic activity which is due to the fact that lignin adsorbs enzyme physically and restricts the physical contact between enzyme and polysaccharides to a large extent [16]. On the contrary, the cell walls of pulped fibres can be rather easily accessed [23], therefore it was speculated that the lignin incrustation predominately inhibits the enzyme activity on cellulose and hemicelluloses [24].

We succeeded in modifying the entirely lignified cell wall of individual fibres by adding a swelling procedure prior to enzyme treatment [15] and by using a high enzyme concentration. Swelling enhanced penetration of enzymes by loosening of polymers or possibly breaking some bonds between hemicelluloses and lignin. The data indicate that the swelling agent itself did not change the mechanical performance of the load-bearing network under tensile loads.

Being a hydrolase, cellulase enzyme was expected to act on $\beta-1-4$-glycosidic linkage of pure cellulose [16]. However, the mode of action might be different in case of native tissues and in pure cellulose. This is due to the higher complexity in the cell wall and presence of other macromolecules like lignin [13]. The mechanisms of enzymatic hydrolysis on crystalline cellulose are not fully clear yet, but hydrogen bond breaking due to action of cellulase on crystalline cellulose was proposed [25, 26]. In agreement with this interpretation, previously Simon and Cosgrove [27] observed that some hydrogen bond breaking within cellulose enhances the enzyme activity.

As FT-IR measurements before and after the enzymatic treatment were done exactly on the same fibre, the observed diminishing of the bands is due to enzymatic treatment. Hence, the reduction of all the bands might be explained by a thinning of the fibre, where all components were partly "excised" as reported by White and Brown [28] for the thinning of bacterial cellulose due to cellulase treatment. Recently, similar thinning of cellulose films due to cellulase activity was reported [29]. Though enzymes are substrate specific, our FT-IR results show some degree of removal of glucomannan and lignin after enzyme 
treatment. This might be explained by the close association between lignin and xylan and between glucomannan and cellulose [30].

Due to enzyme treatment, mechanical behaviour of isolated fibres differed from unmodified fibres and from simply swollen fibres. A reduced ultimate stress level in enzymatic treated fibres revealed the expected mechanical function of the cellulose reinforcement in the entire cell wall. However, only $20 \%$ of the fibres showed the targeted increase in deformability, whereas $80 \%$ of the fibres revealed brittle fracture after the same enzyme treatment. In the mechanical response to the enzyme treated fibres two fracture processes may coexist, due to heterogeneity within the fibre or different enzymatic permeability along the fibre. On one hand, a high number of cellulose fibril bundles are disrupted perpendicular to the cellulose chain direction resulting in a brittle failure event before a gliding of fibrils may occur. On the other hand, even though cellulose fibril bundles are partly disrupted, fibrils start gliding due to a pronounced reduction in cross-linking between cellulose fibrils and matrix. This large deformability of fibres up to $20 \%$ indicates that a partial degradation of cellulose may enable cellulose fibril gliding in normal wood cells, so far only known for wood with high cellulose microfibril angle.

In contrast to the compression wood fibre (Fig. 1), the enzymatically treated normal wood fibres showed severe damage before the plastic deformation indicating different types of deformation mechanisms. The high cellulose microfibril angle in compression wood fibres allows for a gliding of the cellulose fibrils after the shear strength of the matrix is exceeded. Unloading and reloading of the fibres results in a stiffness recovery indicating that the plastic deformation is mainly mediated by the opening of hydrogen bonds, which does not result in a substantial damage [1]. On the contrary, in terms of the enzymatically modified normal wood fibres, the interface between cellulose fibrils and matrix is pre-damaged, which results in high plastic deformation without stiffness recovery most likely due to gliding of fibrils after severe fracture events at the fibril/ matrix interface.

In terms of veneer utilization, the significant reduction of strength and stiffness would be tolerable, since mainly optical properties and a high plastic deformability are important for the covering layer. However, for processing of the veneers, the damage occurring before plastic deformation has to be diminished to avoid too severe or fatal fracture. This problem might be solved by preferential degradation of hemicelluloses, which tether the cellulose fibrils [8]. Another important factor for a transfer of the findings in single fibre tests to veneer processing is related to cell-cell interactions in the veneer. Adjacent cells might reduce the plastic deformability to some extent. However, under wet condition, a higher deformability of individual cells is still preserved in thin tissue sheets ([1] compression wood behaviour) so that cell-cell interactions do not necessarily reduce deformability drastically. Alternatively, the cell-cell interactions might compensate for the fatal fracture of some individual cell members helping to maintain the structural integrity of the veneer.

\section{Conclusion}

In this study, we approached to modify the mechanical performance of individual fibres, while retaining the integrity of the entire cell walls. Microtensile tests on individual fibres showed that the cell wall alteration resulted in a pronounced deformation for two of ten of the modified fibres. The underlying mechanisms for this specific behaviour are still to be examined, but it is assumed that mainly a modification of the amorphous cellulose chains at the surface of the microfibrils may loosen the contact between cellulose fibrils and the pliant matrix. In consequence, cellulose fibrils may start to glide after a critical stress is exceeded making such modified wood material eligible to large deformations. Such a material behaviour would be desirable for covering complex threedimensional geometries with wood veneers. Further studies are needed to optimize the modification at the individual cell level in order to achieve a larger number of highly deformable fibres and to transfer the findings to veneer processing.

Acknowledgements This work was supported by the "Fonds zur Förderung der wissenschaftlichen Forschung (FWF)" and an author (Notburga Gierlinger) by the APART programme of the Austrian Academy of Sciences. The authors like to thank Dr. B. Hinterstoisser, Prof. D. Haltrich and Dr. C. Felby for support at early stages of the project.

\section{References}

1. Keckes J, Burgert I, Frühmann K, Müller M, Kölln K, Hamilton $M$ et al (2003) Nat Mater 2:810

2. Fratzl P, Burgert I, Gupta HS (2004) Phys Chem Chem Phys 6:5575

3. Nassif N, Pinna N, Gehrke N, Antonietti M, Jäger C, Cölfen H (2005) Proc Natl Acad Sci USA 102:12653

4. Aizenberg J, Weaver JC, Thanawala MS, Sundar VC, Morse DE, Fratzl P (2005) Science 309:275

5. Vincent JFV (1990) Structural biomaterials. Princeton University Press, Princeton, New Jersey

6. Vincent JFV (2002) Materials Today 5:28

7. Jeronimidis G (2000) In: Elices M (ed) Structural biological materials-design and structure-property relationships. Pergamon, Amsterdam, The Netherlands, p 3

8. Fahlen J, Salmén L (2005) Biomacromolecules 6:433

9. Cave ID, Walker JCF (1994) Forest Prod J 44:43 
10. Lichtenegger H, Reiterer A, Tschegg S, Fratzl P (1998) In: Butterfield BG (ed) Microfibril angle in wood. IAWA-Press, p 140

11. Reiterer A, Lichtenegger H, Tschegg S, Fratzl P (1999) Philos Mag A 79:2173

12. Wagenführ A, Buchelt B, Pfriem A (2006) Holz als Roh- und Werkstoff 64:83

13. Rosenthal M, Wagenführ A, Burgert I, Speck T (2006) In: Salmén L (ed) Proceedings of the 5th plant biomechanics conference. Stockholm, Sweden, p 89

14. Burgert I, Gierlinger N, Zimmermann T (2005) Holzforschung 59:240

15. Chen JY, Shimiju Y, Takai M, Hayashi J (1995) J Wood Sci Technol 29:295

16. Huang AA (1975) Biotechnol Bioeng Symp 5:245

17. Burgert I, Frühmann K, Keckes J, Fratzl P, Stanzl-Tschegg SE (2003) Holzforschung 57:661

18. Fengel D (1993) Holzforschung 47:103

19. Liang CY, Marchessault RH (1959) J Polym Sci 39:269
20. Liang CY, Basset KH, McGinnes EA, Marchessault RH (1960) Tappi 43:1017

21. Marchessault RH (1962) Pure Appl Chem 5:107

22. Hergert HL (1971) In: Sarkanen KV, Ludwig CH (eds) Lignins. Occurrence, formation, structure and reactions. John Wiley \& Sons, New York, Chichester, Brisbane, Toronto, Singapore, p 267

23. Hilden L (2004) Doctoral thesis. Department of Wood Science, Swedish University of Agricultural Sciences, Uppsala

24. Polcin J, Bezuch B (1977) Wood Sci Technol 11:275

25. Peiji G, Jie L, Yuzhong Z, Yinbo Q, Shijin P (1998) Prog Nat Sci $8: 117$

26. Gao PJ, Chen GJ, Wang TH, Zhang YS, Liu J (2001) Acta Biochim Biophys Sinica 33:13

27. Simon MM, Cosgrove DJ (1994) Proc Natl Acad Sci USA 91:6574

28. White AR, Brown RM (1981) Proc Natl Acad Sci USA 78:1047

29. Eriksson J, Malmsten M, Tiberg F, Callisen TH, Damhus T, Johansen KS (2005) J Colloid Interf Sci 284:99

30. Salmén L, Olsson AM (1998) J Pulp Paper Sci 24:99 